\title{
Refinements of the bottom and strange MSSM Higgs Yukawa couplings at NNLO
}

\author{
Margherita Ghezzi $^{1}$, Seraina Glaus ${ }^{2,3}$, Dario Müller ${ }^{4}$, Timo Schmidt ${ }^{5}$, Michael Spira ${ }^{6, a}$ \\ ${ }^{1}$ Institut für Theoretische Physik, Eberhard Karls, Universität Tübingen, 72076, Tübingen, Germany \\ ${ }^{2}$ Institute for Theoretical Physics, Karlsruhe Institute of Technology, 76131 Karlsruhe, Germany \\ ${ }^{3}$ Institute for Nuclear Physics, Karlsruhe Institute of Technology, 76344 Karlsruhe, Germany \\ ${ }^{4}$ Institut für Theoretische Physik, Zurich University, CH-8057 Zurich, Switzerland \\ ${ }_{6}^{5}$ Physikalisches Institut, Albert-Ludwigs-Universität Freiburg, 79104 Freiburg, Germany \\ ${ }^{6}$ Paul Scherrer Institut, CH-5232 Villigen PSI, Switzerland
}

Received: 14 July 2020 / Accepted: 8 March 2021 / Published online: 25 March 2021

(C) The Author(s) 2021

\begin{abstract}
We extend the already existing two-loop calculation of the effective bottom-Yukawa coupling in the MSSM. In addition to the resummation of the dominant corrections for large values of $\operatorname{tg} \beta$, we include the subleading terms related to the trilinear Higgs coupling $A_{b}$ and contributions induced by the electroweak gauge couplings. This calculation has been extended to the NNLO corrections to the MSSM strange-Yukawa coupling. Our analysis leads to residual theoretical uncertainties of the effective Yukawa couplings at the per-cent level.
\end{abstract}

\section{Introduction}

The discovery of a Standard-Model-like Higgs boson at the LHC [1,2] completed the theory of electroweak and strong interactions. The existence of an elementary Higgs boson [3$8]$ is a necessary ingredient of a weakly interacting renormalizable theory with spontaneous symmetry breaking $[9,10]$. The measured Higgs mass of (125.09 \pm 0.24$) \mathrm{GeV}$ [11] ranges at the order of the weak scale. However, if embedded in a Grand Unified Theory (GUT), radiative corrections tend to push the Higgs mass towards the GUT scale, if the Higgs couples to particles at this large scale. This problem is known as the hierarchy problem [12-15]. A solution to this problem might be offered by supersymmetry (SUSY) at the TeV scale [16-22].

The minimal supersymmetric extension of the Standard Model (MSSM) contains two Higgs doublets yielding five elementary Higgs bosons after electroweak symmetry breaking, two neutral CP-even (scalar) bosons $h, H$, one neutral CP-odd (pseudoscalar) boson $A$ and two charged bosons

a e-mail: michael.spira@psi.ch (corresponding author)
$H^{ \pm}$. The MSSM Higgs sector is described by two input parameters at leading order, which are generally chosen as $\operatorname{tg} \beta=v_{2} / v_{1}$, the ratio of the two vacuum expectation values $v_{1,2}$, and the pseudoscalar Higgs mass $M_{A}$, if all SUSY parameters are real. Including the one-loop and leading twoloop corrections, the upper bound on the light scalar Higgs mass is lifted to $M_{h} \lesssim 130 \mathrm{GeV}$ if stop masses are constrained to less than $10 \mathrm{TeV}$ [23-28]. The more recent threeloop results modify this upper bound by less than $1 \mathrm{GeV}$ [29-31]. The Higgs couplings to gauge bosons and fermions involve mixing angles $\alpha$ and $\beta$, which are defined by diagonalizing the neutral and charged Higgs mass matrices. They are listed in Table 1 relative to the SM Higgs couplings.

For large $\operatorname{tg} \beta$ values the down-type Yukawa couplings are enhanced and the up-type Yukawa couplings suppressed, if the light (heavy) scalar Higgs mass does not range at its upper (lower) bound, where the couplings become StandardModel-like (up to a sign for the heavy scalar Higgs boson) ${ }^{1}$. At present and future colliders this property leads to the dominance of bottom-Yukawa-coupling induced heavy-Higgs processes for large $\operatorname{tg} \beta$ values as heavy Higgs decays into bottom quarks and heavy Higgs bremsstrahlung off bottom quarks at hadron and $e^{+} e^{-}$colliders. In addition, Higgsboson production via gluon fusion $g g \rightarrow H, A$ is dominantly induced by the bottom-loop contributions for large $\operatorname{tg} \beta$. The strongly enhanced strange-Yukawa coupling on the other hand plays a role for the subleading charged Higgs decay mode $H^{+} \rightarrow c \bar{s}$ that can reach branching ratios at the per-cent level or the reverse charged Higgs production process $c \bar{s} \rightarrow H^{+}[33]$.

\footnotetext{
${ }^{1}$ Scenarios with a SM-like heavy scalar Higgs boson are disfavoured by the present searches [32].
} 
Table 1 MSSM Higgs couplings to SM particles relative to the SM Higgs couplings

\begin{tabular}{lllll}
\hline$\Phi$ & & $g_{u}^{\Phi}$ & $g_{d}^{\Phi}$ & $g_{V}^{\Phi}$ \\
\hline SM & $H$ & 1 & 1 & 1 \\
MSSM & $h$ & $\cos \alpha / \sin \beta$ & $-\sin \alpha / \cos \beta$ & $\sin (\beta-\alpha)$ \\
& $H$ & $\sin \alpha / \sin \beta$ & $\cos \alpha / \cos \beta$ & $\cos (\beta-\alpha)$ \\
& $A$ & $1 / \operatorname{tg} \beta$ & $\operatorname{tg} \beta$ & 0 \\
\hline
\end{tabular}

The soft SUSY-breaking terms in the MSSM induce mixing of the current sfermion eigenstates $\tilde{f}_{L}$ and $\tilde{f}_{R}$. The sfermion mass matrix in the current eigenstate basis is given by $^{2}$

$$
\begin{aligned}
\mathcal{M}_{\tilde{f}}^{2} & =\left(\begin{array}{cc}
M_{L L}^{2} M_{L R}^{2} \\
M_{R L}^{2} M_{R R}^{2}
\end{array}\right) \\
& =\left(\begin{array}{cc}
M_{\tilde{f}_{L}}^{2}+m_{f}^{2} & m_{f}\left(A_{f}-\mu r_{f}\right) \\
m_{f}\left(A_{f}-\mu r_{f}\right) & M_{\tilde{f}_{R}}^{2}+m_{f}^{2}
\end{array}\right)
\end{aligned}
$$

with the factors $r_{d}=1 / r_{u}=\operatorname{tg} \beta$ for down- and uptype sfermions. The parameter $\mu$ denotes the higgsino mass parameter of the superpotential and $A_{f}$ the soft SUSYbreaking trilinear couplings. The sfermion mass eigenstates $\tilde{f}_{1,2}$ emerge from the current eigenstates $\tilde{f}_{L, R}$ through rotations by mixing angles $\theta_{f}$,

$\tilde{f}_{1}=\tilde{f}_{L} \cos \theta_{f}+\tilde{f}_{R} \sin \theta_{f}$

$\tilde{f}_{2}=-\tilde{f}_{L} \sin \theta_{f}+\tilde{f}_{R} \cos \theta_{f}$

which scale with the masses $m_{f}$ of the SM fermions. Mixing effects are only relevant for the third-generation sfermions $\tilde{t}, \tilde{b}, \tilde{\tau}$ and will thus be neglected for the strange squarks in this work. The mixing angles are determined by

$$
\begin{aligned}
\sin 2 \theta_{f} & =\frac{2 m_{f}\left(A_{f}-\mu r_{f}\right)}{m_{\tilde{f}_{1}}^{2}-m_{\tilde{f}_{2}}^{2}}, \\
\cos 2 \theta_{f} & =\frac{M_{\tilde{f}_{L}}^{2}-M_{\tilde{f}_{R}}^{2}}{m_{\tilde{f}_{1}}^{2}-m_{\tilde{f}_{2}}^{2}}
\end{aligned}
$$

while the masses of the squark mass eigenstates read as

$$
\begin{aligned}
m_{\tilde{f}_{1,2}}^{2}= & m_{f}^{2}+\frac{1}{2}\left[M_{\tilde{f}_{L}}^{2}\right. \\
& \left.+M_{\tilde{f}_{R}}^{2} \mp \sqrt{\left(M_{\tilde{f}_{L}}^{2}-M_{\tilde{f}_{R}}^{2}\right)^{2}+4 m_{f}^{2}\left(A_{f}-\mu r_{f}\right)^{2}}\right]
\end{aligned}
$$

The topic of this work is the extension of the next-tonext-to-leading-order (NNLO) SUSY-QCD and top-induced SUSY-electroweak corrections of the effective bottomYukawa couplings $[34,35]$ to the terms induced by the soft

\footnotetext{
${ }^{2}$ For convenience, the $D$-terms have been absorbed in the soft SUSYbreaking sfermion mass parameters $M_{\tilde{f}_{L / R}}^{2}$.
}

SUSY-breaking trilinear coupling $A_{b}$, the electroweak couplings $\alpha_{1}=g^{\prime 2} /(4 \pi), \alpha_{2}=g^{2} /(4 \pi)\left[g, g^{\prime}\right.$ being the isospin and hypercharge gauge couplings, respectively] and to the SUSY-QCD corrections of the strange-Yukawa couplings. The results will play a role in all processes to which the bottom- and strange-Yukawa couplings contribute. In particular the neutral and charged Higgs decay widths and Higgs radiation off bottom quarks at hadron colliders, which constitutes the dominant heavy Higgs boson production channel for large $\operatorname{tg} \beta$ at the LHC [36-38], are affected.

\section{Effective bottom- and strange-Yukawa couplings}

The dominant parts of the SUSY-QCD (and SUSYelectroweak) corrections to processes mediated by the bottom- and strange-Yukawa couplings can be described in terms of effective bottom- and strange-Yukawa couplings. These corrections arise in the limit of heavy supersymmetric particles relative to the energy scale of the particular process. The reliability of this large mass approximation has been analyzed for neutral MSSM Higgs decays into bottom quarks $h / H / A \rightarrow b \bar{b}$ [39], charged Higgs decays to top and bottom quarks $H^{ \pm} \rightarrow t b[40]$ and Higgs radiation off bottom quarks at $e^{+} e^{-}$colliders [41,42] and hadron colliders [4347] by comparing to the full next-to-leading-order (NLO) results. For large values of $\operatorname{tg} \beta$ the approximation agrees with the NLO results at the sub-per-cent level for SUSY masses in the $\mathrm{TeV}$ range.

\subsection{Effective Lagrangian}

The dominant contributions to the MSSM bottom- and strange-Yukawa couplings can be obtained from the effective Lagrangian $[39,40]$

$$
\begin{aligned}
\mathcal{L}_{e f f}= & -\sum_{q=b, s} \lambda_{q} \overline{q_{R}}\left[\left(1+\Delta_{q, 1}\right) \phi_{1}^{0}+\Delta_{q, 2} \phi_{2}^{0 *}\right] q_{L}+\text { h.c. } \\
= & -\sum_{q=b, s} m_{q} \bar{q}\left[1+i \gamma_{5} \frac{G^{0}}{v}\right] q \\
& -\frac{m_{q} / v}{1+\Delta_{q}} \bar{q}\left[g_{q}^{h}\left(1-\frac{\Delta_{q}}{\operatorname{tg} \alpha \operatorname{tg} \beta}\right) h\right. \\
& \left.+g_{q}^{H}\left(1+\Delta_{q} \frac{\operatorname{tg} \alpha}{\operatorname{tg} \beta}\right) H-g_{q}^{A}\left(1-\frac{\Delta_{q}}{\operatorname{tg}^{2} \beta}\right) i \gamma_{5} A\right] q
\end{aligned}
$$

with the individual leading one-loop terms for the bottom Yukawa couplings $\left(C_{F}=4 / 3\right)[48-50]$

$$
\begin{aligned}
\Delta_{b, 1} & =-\frac{C_{F}}{2} \frac{\alpha_{s}\left(\mu_{R}\right)}{\pi} m_{\tilde{g}} A_{b}\left(\mu_{R}\right) I\left(m_{\tilde{b}_{1}}^{2}, m_{\tilde{b}_{2}}^{2}, m_{\tilde{g}}^{2}\right) \\
\Delta_{b, 2} & =\Delta_{b, 2}^{Q C D}+\Delta_{b, 2}^{e l w, t}+\Delta_{b, 2}^{e l w, 1}+\Delta_{b, 2}^{e l w, 2}
\end{aligned}
$$




$$
\begin{aligned}
\Delta_{b, 2}^{Q C D}= & \frac{C_{F}}{2} \frac{\alpha_{s}\left(\mu_{R}\right)}{\pi} m_{\tilde{g}} \mu I\left(m_{\tilde{b}_{1}}^{2}, m_{\tilde{b}_{2}}^{2}, m_{\tilde{g}}^{2}\right) \\
\Delta_{b, 2}^{e l w, t}= & \frac{\lambda_{t}^{2}\left(\mu_{R}\right)}{(4 \pi)^{2}} A_{t}\left(\mu_{R}\right) \mu I\left(m_{\tilde{t}_{1}}^{2}, m_{\tilde{t}_{2}}^{2}, \mu^{2}\right) \\
\Delta_{b, 2}^{e l w, 1}= & -\frac{\alpha_{1}}{12 \pi} M_{1} \mu \\
& \left\{\frac{1}{3} I\left(m_{\tilde{b}_{1}}^{2}, m_{\tilde{b}_{2}}^{2}, M_{1}^{2}\right)+\left(\frac{c_{b}^{2}}{2}+s_{b}^{2}\right) I\left(m_{\tilde{b}_{1}}^{2}, M_{1}^{2}, \mu^{2}\right)\right. \\
& \left.+\left(\frac{s_{b}^{2}}{2}+c_{b}^{2}\right) I\left(m_{\tilde{b}_{2}}^{2}, M_{1}^{2}, \mu^{2}\right)\right\} \\
\Delta_{b, 2}^{e l w, 2}= & -\frac{\alpha_{2}}{4 \pi} M_{2} \mu\left\{c_{t}^{2} I\left(m_{\tilde{t}_{1}}^{2}, M_{2}^{2}, \mu^{2}\right)\right. \\
& +s_{t}^{2} I\left(m_{\tilde{t}_{2}}^{2}, M_{2}^{2}, \mu^{2}\right) \\
& \left.+\frac{c_{b}^{2}}{2} I\left(m_{\tilde{b}_{1}}^{2}, M_{2}^{2}, \mu^{2}\right)+\frac{s_{b}^{2}}{2} I\left(m_{\tilde{b}_{2}}^{2}, M_{2}^{2}, \mu^{2}\right)\right\}
\end{aligned}
$$

and for the strange-Yukawa couplings ${ }^{3}$

$$
\begin{aligned}
\Delta_{s, 1}= & -\frac{C_{F}}{2} \frac{\alpha_{s}\left(\mu_{R}\right)}{\pi} m_{\tilde{g}} A_{s}\left(\mu_{R}\right) I\left(m_{\tilde{s}_{1}}^{2}, m_{\tilde{s}_{2}}^{2}, m_{\tilde{g}}^{2}\right) \\
\Delta_{s, 2}= & \Delta_{s, 2}^{Q C D}+\Delta_{s, 2}^{e l w, 1}+\Delta_{s, 2}^{e l w, 2} \\
\Delta_{s, 2}^{Q C D}= & \frac{C_{F}}{2} \frac{\alpha_{s}\left(\mu_{R}\right)}{\pi} m_{\tilde{g}} \mu I\left(m_{\tilde{s}_{1}}^{2}, m_{\tilde{s}_{2}}^{2}, m_{\tilde{g}}^{2}\right) \\
\Delta_{s, 2}^{e l w}= & -\frac{\alpha_{1}}{12 \pi} M_{1} \mu\left\{\frac{1}{3} I\left(m_{\tilde{s}_{1}}^{2}, m_{\tilde{s}_{2}}^{2}, M_{1}^{2}\right)\right. \\
& +\left(\frac{c_{s}^{2}}{2}+s_{s}^{2}\right) I\left(m_{\tilde{s}_{1}}^{2}, M_{1}^{2}, \mu^{2}\right) \\
& \left.+\left(\frac{s_{s}^{2}}{2}+c_{s}^{2}\right) I\left(m_{\tilde{s}_{2}}^{2}, M_{1}^{2}, \mu^{2}\right)\right\} \\
\Delta_{s, 2}^{e l w, 2}= & -\frac{\alpha_{2}}{4 \pi} M_{2} \mu\left\{c_{c}^{2} I\left(m_{\tilde{c}_{1}}^{2}, M_{2}^{2}, \mu^{2}\right)\right. \\
& +s_{c}^{2} I\left(m_{\tilde{c}_{2}}^{2}, M_{2}^{2}, \mu^{2}\right) \\
& \left.+\frac{c_{s}^{2}}{2} I\left(m_{\tilde{s}_{1}}^{2}, M_{2}^{2}, \mu^{2}\right)+\frac{s_{s}^{2}}{2} I\left(m_{\tilde{s}_{2}}^{2}, M_{2}^{2}, \mu^{2}\right)\right\}
\end{aligned}
$$

where $s_{q}=\sin \theta_{q}, c_{q}=\cos \theta_{q}(q=t, b, c, s)$ are related to the squark mixing angles $\theta_{q}$ of Eq. (3). The final contribution in the mass-eigenstate-basis can be derived as

$\Delta_{q}=\frac{\Delta_{q, 2} \operatorname{tg} \beta}{1+\Delta_{q, 1}} \quad(q=b, s)$

where the $\Delta_{q, 1}$ terms have been shown to be properly resummed in this way [39] $]^{4}$. The auxiliary function $I$ is given

\footnotetext{
${ }^{3}$ Due to the tiny charm-Yukawa coupling $\lambda_{c}$ we neglect electroweak corrections induced by $\lambda_{c}$. Moreover, for the strange and charm squarks we neglect mixing effects, i.e. work with $c_{s / c}^{2}=1$ and $s_{s / c}^{2}=0$.

4 The $A_{b, s}$ terms in the resummed expression of Eq. (8) are formally of three-loop order and thus subleading compared to the $\Delta_{q, 2}$ contributions. Electroweak contributions to $\Delta_{q, 1}$ have therefore been neglected, since these are expected to be phenomenologically irrelevant.
}

by

$I(a, b, c)=\frac{a b \log \frac{a}{b}+b c \log \frac{b}{c}+c a \log \frac{c}{a}}{(a-b)(b-c)(a-c)}$

The field amplitudes $\phi_{1}^{0}$ and $\phi_{2}^{0}$ of the neutral components of the Higgs doublets that couple to down- and up-type quarks, respectively, are transformed to the mass eigenstates $h, H, A$ by the mixing angles $\alpha, \beta$

$\phi_{1}^{0}=\frac{1}{\sqrt{2}}\left[v_{1}+H \cos \alpha-h \sin \alpha+i A \sin \beta-i G^{0} \cos \beta\right]$
$\phi_{2}^{0}=\frac{1}{\sqrt{2}}\left[v_{2}+H \sin \alpha+h \cos \alpha+i A \cos \beta+i G^{0} \sin \beta\right]$

The two vacuum expectation values are connected to the Fermi constant $G_{F}$ by $v^{2}=v_{1}^{2}+v_{2}^{2}=1 /\left(\sqrt{2} G_{F}\right)$. The would-be Goldstone field $G^{0}$ is 'eaten' by the $Z$ boson and builds up its longitudinal component. The top-Yukawa coupling $\lambda_{t}$ determines the top mass by $m_{t}=\lambda_{t} v_{2} / \sqrt{2}$ at leading order. The soft SUSY-breaking trilinear couplings of the top, bottom and strange squarks are denoted by $A_{t}, A_{b}$ and $A_{s}$, the higgsino mass parameter by $\mu$ and the strong coupling constant by $\alpha_{s}$. The renormalization scale is depicted as $\mu_{R}$. The corrections $\Delta_{b, s}$ modify the relation between the bottom (strange) quark mass $m_{b}\left(m_{s}\right)$ and the bottom (strange) Yukawa coupling $\lambda_{b}\left(\lambda_{S}\right)$,

$m_{q}=\frac{\lambda_{q} v_{1}}{\sqrt{2}}\left[1+\Delta_{q, 1}+\Delta_{q, 2} \operatorname{tg} \beta\right] \quad(q=b, s)$

The effective Lagrangian of Eq. (5) can be expressed as (omitting the mass and Goldstone terms)

$\mathcal{L}_{e f f}=-\sum_{q=b, s} \frac{m_{q}}{v} \bar{q}\left[\tilde{g}_{q}^{h} h+\tilde{g}_{q}^{H} H-\tilde{g}_{q}^{A} i \gamma_{5} A\right] q$

with the effective (resummed) couplings

$\tilde{g}_{q}^{h}=\frac{g_{q}^{h}}{1+\Delta_{q}}\left[1-\frac{\Delta_{q}}{\operatorname{tg} \alpha \operatorname{tg} \beta}\right]$

$\tilde{g}_{q}^{H}=\frac{g_{q}^{H}}{1+\Delta_{q}}\left[1+\Delta_{q} \frac{\operatorname{tg} \alpha}{\operatorname{tg} \beta}\right]$

$\tilde{g}_{q}^{A}=\frac{g_{q}^{A}}{1+\Delta_{q}}\left[1-\frac{\Delta_{q}}{\operatorname{tg} \beta^{2}}\right]$

Even though the SUSY corrections $\Delta_{q}$ are loop-suppressed, they are significant for large values of $\operatorname{tg} \beta$. In these regions they dominate the supersymmetric corrections to the bottomand strange-Yukawa couplings. The effective Lagrangian in Eq. (5) has been derived by integrating out the heavy SUSY particles so that it is not only valid for large values of $\operatorname{tg} \beta$. By using power counting it has been shown that the Lagrangian of Eq. (5) resums all terms of $\mathcal{O}\left[\left(\alpha_{s} \mu \operatorname{tg} \beta\right)^{n}\right]$ and 

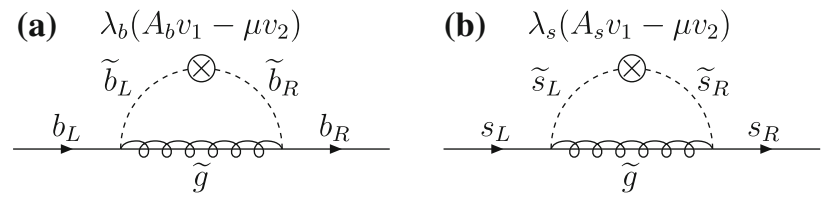

Fig. 1 One-loop diagrams of the SUSY-QCD contributions to a the bottom and $\mathbf{b}$ the strange self-energies with the off-diagonal mass insertions related to the $\Delta_{q}(q=b, s)$ corrections of the bottom- and strange-Yukawa couplings. The contributing particles involve bottom and strange quarks $b, s$ and squarks $\tilde{b}, \tilde{s}$ as well as gluinos $\widetilde{g}$

$\mathcal{O}\left[\left(\alpha_{s} A_{b, s}\right)^{n}\right]$. For the bottom-Yukawa coupling a resummation of the $\mathcal{O}\left[\left(\lambda_{t}^{2} A_{t} \operatorname{tg} \beta\right)^{n}\right]$ terms is achieved in addition $[39,40]$ (including mixed contributions).

\subsection{Low energy theorems}

The derivation of higher-order corrections to the effective Yukawa couplings would need the calculation of the related three-point functions in the low-energy limit. This, however, can be reduced to the determination of self-energy diagrams by means of low energy theorems [51-55]. These are based on the feature that in the limit of vanishing Higgs momentum, matrix elements with an external Higgs boson can be generated from the corresponding matrix elements without the external Higgs particle by the shifts $v_{1} \rightarrow \sqrt{2} \phi_{1}^{0}$ and $v_{2} \rightarrow \sqrt{2} \phi_{2}^{0 *}$. Thus, only the calculation of the related parts of the bottom and strange quark self-energies is required. The dominant parts $\Delta_{q, 1 / 2}(q=b, s)$ originate from the scalar part $\Sigma_{S}\left(m_{q}^{2}\right)$ of the self-energy ${ }^{5}$. This affects the relation between the bottom- (strange-) Yukawa coupling $\lambda_{b}\left(\lambda_{s}\right)$ and the mass $m_{q}$ of the bottom (strange) quark,

$m_{q}=\frac{\lambda_{q}}{\sqrt{2}} v_{1}+\Sigma_{S}\left(m_{q}^{2}\right)$

with the dominant terms of the self-energy $\Sigma_{S}\left(m_{q}^{2}\right)$ for heavy SUSY particles

$\Sigma_{S}\left(m_{q}^{2}\right)=\frac{\lambda_{q}}{\sqrt{2}} v_{1}\left[\Delta_{q, 1}+\Delta_{q, 2} \operatorname{tg} \beta\right]$

The NLO-QCD parts of $\Delta_{b}$ and $\Delta_{s}$ in Eq. (5) can be obtained from off-diagonal mass insertions of the type $\lambda_{q}\left(A_{q} v_{1}-\mu v_{2}\right)$ (up to a factor $1 / \sqrt{2}$ ) in the squark propagators, as shown in Fig. 1 at one-loop order.

This results in the finite expressions of Eqs. $(6,7)$ (supplemented by the SUSY-electroweak corrections originating from higgsino, wino and bino exchange to the bottomYukawa couplings) after transforming the fields from currenteigenstates to the mass eigenstates. These contributions are

\footnotetext{
5 The fermionic self-energy can be split into a scalar, vectorial and axial-vectorial part as $\Sigma(p)=\Sigma_{S}\left(p^{2}\right)+\not p \Sigma_{V}\left(p^{2}\right)+\not p \gamma_{5} \Sigma_{A}\left(p^{2}\right)$.
}

not renormalized at NLO due to the absence of tree-level bottom and strange couplings involving $A_{q}$ or $\mu$.

\section{NNLO corrections}

The NNLO analysis of the effective bottom- and strangeYukawa couplings necessitates the calculation of the leading NNLO corrections to the bottom and strange self-energies. The NNLO expressions of the bottom-Yukawa couplings have been obtained in Refs. [34,35,56-58]. We will extend these results to the non- $\operatorname{tg} \beta$-enhanced $A_{b}$ terms and to the strange Yukawa couplings. In this work we will neglect intergenerational mixing so that issues related to the flavour sector [59-65] can be disregarded.

\subsection{Bottom-Yukawa couplings}

\subsubsection{SUSY-QCD corrections to $\Delta_{b, 1}$}

Since the effective insertions according to Fig. 1 are always proportional to $A_{b}-\mu \operatorname{tg} \beta$, the contributions of all two-loop diagrams for the bottom-Yukawa coupling are the same for the $A_{b}$ and the $\mu \operatorname{tg} \beta$ contributions (up to the relative overall sign). The renormalization proceeds along the lines of Ref. $[34,35]$ so that the SUSY-QCD corrections to the $\Delta_{b, 1}$ terms are the same as for the $\Delta_{b, 2}$ contributions after renormalization (including the SUSY-restoring counter terms [66]). Denoting the (renormalized) NNLO-corrected SUSY-QCD part $\Delta_{b, 2}^{Q C D}$ of Refs. $[34,35]$ as

$\Delta_{b, 2}^{Q C D}=\mu \Delta^{N L O}\left[1+\delta_{b}\right]$

with the NNLO correction $\delta_{b}$ and

$\Delta^{N L O}=\frac{C_{F}}{2} \frac{\alpha_{s}\left(\mu_{R}\right)}{\pi} m_{\tilde{g}} I\left(m_{\tilde{b}_{1}}^{2}, m_{\tilde{b}_{2}}^{2}, m_{\tilde{g}}^{2}\right)$

the effective correction to the bottom-Yukawa couplings of Eq. (8) acquires the form

$$
\begin{aligned}
& \Delta_{b}= \\
& \frac{\mu \Delta^{N L O}\left[1+\delta_{b}\right]+\Delta_{b, 2}^{e l w, t}\left[1+\delta_{t}\right]+\Delta_{b, 2}^{e l w, 1}\left[1+\delta_{1}\right]+\Delta_{b, 2}^{e l w, 2}\left[1+\delta_{2}\right]}{1-A_{b}^{0} \Delta^{N L O}\left[1+\delta_{b}\right]} \operatorname{tg} \beta
\end{aligned}
$$

where $A_{b}^{0}$ denotes the bare trilinear coupling that is renormalized in SUSY-QCD and $\delta_{t}$ the SUSY-QCD corrections to $\Delta_{b, 2}^{e l w, t}[34,35]$. The NNLO SUSY-QCD corrections $\delta_{1}$ $\left(\delta_{2}\right)$ to $\Delta_{b, 2}^{e l w, 1}\left(\Delta_{b, 2}^{e l w, 2}\right)$ will be derived and discussed in the next subsection. The renormalization of $A_{b}$ emerges from a non-leading order contribution in our context: for the $\overline{\mathrm{MS}}$ renormalized trilinear coupling within dimensional regularization in $n=4-2 \epsilon$ dimensions we obtain 


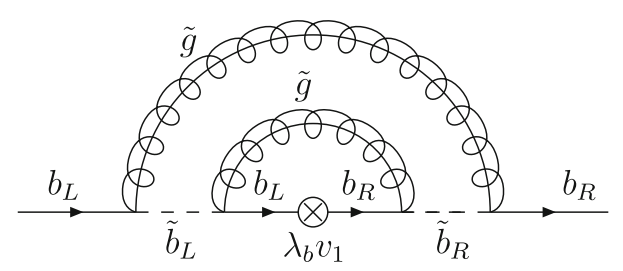

Fig. 2 Two-loop diagram of sbottom-self-energy insertions contributing to the SUSY-QCD corrections to the bottom-quark self-energy. This involves bottom quarks $b$, bottom squarks $\tilde{b}$ and gluinos $\tilde{g}$

$$
\begin{aligned}
A_{b}^{0} & =A_{b}\left(\mu_{R}^{2}\right)+\delta A_{b} \\
\delta A_{b} & =C_{F} \frac{\alpha_{s}}{\pi} \Gamma(1+\epsilon)\left(\frac{4 \pi \mu^{2}}{\mu_{R}^{2}}\right)^{\epsilon} \frac{m_{\tilde{g}}}{\epsilon} \neq \mathcal{O}\left(A_{b}\right)
\end{aligned}
$$

so that $A_{b}$ is not renormalized at $\mathcal{O}\left(\alpha_{s} A_{b}\right)$. We have explicitly checked that the divergence corresponding to the counterterm of $A_{b}$ is generated by the diagram of Fig. 2 with an insertion $\lambda_{b} v_{1}$ (up to a factor $1 / \sqrt{2}$ ) at the virtual bottomquark line. Thus the final expression including the $\mathcal{O}\left(A_{b}\right)$ terms is given by Eq. (18) with $A_{b}^{0}$ replaced by the renormalized $A_{b}\left(\mu_{R}^{2}\right)$ coupling,

$$
\Delta_{b}=\frac{\Delta_{b, 2}^{Q C D}\left[1+\delta_{b}\right]+\Delta_{b, 2}^{e l w, t}\left[1+\delta_{t}\right]+\Delta_{b, 2}^{e l w, 1}\left[1+\delta_{1}\right]+\Delta_{b, 2}^{e l w, 2}\left[1+\delta_{2}\right]}{1+\Delta_{b, 1}\left[1+\delta_{b}\right]} \operatorname{tg} \beta
$$

with $\Delta_{b, 1}, \Delta_{b, 2}^{Q C D}, \Delta_{b, 2}^{e l w, t}, \Delta_{b, 2}^{e l w, 1}$ and $\Delta_{b, 2}^{e l w, 2}$ defined in Eq. (6).

In this work we adopt the renormalization program of Ref. [67], i.e. the counterterm for the top-Yukawa-induced electroweak contributions $\Delta_{b, 2}^{e l w, t}$ is modified for the trilinear coupling $A_{t}$ that is defined in the $\overline{\mathrm{MS}}$ scheme. This leads to a vanishing counterterm for $A_{t}$ at the order we are calculating.

\subsubsection{SUSY-QCD corrections to $\Delta_{b, 2}^{e l w, 1 / 2}$}

For the calculation of the SUSY-QCD corrections to the terms $\Delta_{b, 2}^{e l w, 1}$ and $\Delta_{b, 2}^{e l w, 1}$ we will reduce the associated higgsino propagators to the contributions relevant for the $\operatorname{tg} \beta$-enhanced corrections. For the neutralinos in the basis $\left(\tilde{B}, \tilde{W}^{3}, \tilde{H}_{1}^{0}, \tilde{H}_{2}^{0}\right)$, the full inverse propagator matrix is given by

$$
\begin{aligned}
\mathcal{P}^{-1} & =\mathcal{P}_{0}^{-1}+\mathcal{D} \\
\mathcal{P}_{0}^{-1} & =\left(\begin{array}{ccccc}
\not p-M_{1} & 0 & 0 & 0 \\
0 & \not p-M_{2} & 0 & 0 \\
0 & 0 & \not p & \mu \\
0 & 0 & \mu & \not p
\end{array}\right)
\end{aligned}
$$

$$
\mathcal{D}=\left(\begin{array}{cccc}
0 & 0 & \frac{g^{\prime} v}{2} c_{\beta} & -\frac{g^{\prime} v}{2} s_{\beta} \\
0 & 0 & -\frac{g v}{2} c_{\beta} & \frac{g v}{2} s_{\beta} \\
\frac{g^{\prime} v}{2} c_{\beta} & -\frac{g v}{2} c_{\beta} & 0 & 0 \\
-\frac{g^{\prime} v}{2} s_{\beta} & \frac{g v}{2} s_{\beta} & 0 & 0
\end{array}\right)
$$

where the part $\mathcal{D}$ is subleading in the limit of heavy SUSY particles that we are working in. Keeping only linear terms in $\mathcal{D}$, the propagator matrix is then given by

$$
\begin{aligned}
\mathcal{P}= & \mathcal{P}_{0}-\mathcal{P}_{0} \mathcal{D} \mathcal{P}_{0} \\
\mathcal{P}_{0}= & \left(\begin{array}{cccc}
\frac{\not p+M_{1}}{p^{2}-M_{1}^{2}} & 0 & 0 & 0 \\
0 & \frac{\not p+M_{2}}{p^{2}-M_{2}^{2}} & 0 & 0 \\
0 & 0 & \frac{\not p}{p^{2}-\mu^{2}} & -\frac{\mu}{p^{2}-\mu^{2}} \\
0 & 0 & -\frac{\mu}{p^{2}-\mu^{2}} & \frac{\not p}{p^{2}-\mu^{2}}
\end{array}\right) \\
& -\mathcal{P}_{0} \mathcal{D} \mathcal{P}_{0}=\left(\begin{array}{cc}
0 & \mathcal{A} \\
\mathcal{A}^{T} & 0
\end{array}\right) \\
\mathcal{A} & =\frac{c_{\beta}}{p^{2}-\mu^{2}}\left(\begin{array}{cc}
-\frac{g^{\prime} v}{2} \frac{\left(\not p+M_{1}\right)(\not p+\mu \operatorname{tg} \beta)}{p^{2}-M_{1}^{2}} & \frac{g^{\prime} v}{2} \frac{\left(\not p+M_{1}\right)(\not p \operatorname{tg} \beta+\mu)}{p^{2}-M_{1}^{2}} \\
\frac{g v}{2} \frac{\left(\not p+M_{2}\right)(\not p+\mu \operatorname{tg} \beta)}{p^{2}-M_{2}^{2}} & -\frac{g v}{2} \frac{\left(\not p+M_{2}\right)(\not p \operatorname{tg} \beta+\mu)}{p^{2}-M_{2}^{2}}
\end{array}\right) .
\end{aligned}
$$

Inserting the corresponding diagonal and off-diagonal propagators into the self-energies of Fig. 3 and keeping only the $\operatorname{tg} \beta$-enhanced terms in the numerators of the off-diagonal propagators contained in $\mathcal{A}$, we arrive at the proper expressions for $\Delta_{b, 2}^{e l w, 1}$ of Eq. (6) where the identities

$$
\begin{aligned}
& M_{L L}^{2}=M_{\tilde{q}_{L}}^{2}+m_{q}^{2}=m_{\tilde{q}_{1}}^{2} c_{q}^{2}+m_{\tilde{q}_{2}}^{2} s_{q}^{2} \\
& M_{R R}^{2}=M_{\tilde{q}_{R}}^{2}+m_{q}^{2}=m_{\tilde{q}_{1}}^{2} s_{q}^{2}+m_{\tilde{q}_{2}}^{2} c_{q}^{2} \quad(q=b, t)
\end{aligned}
$$

have been used. Including only the $\operatorname{tg} \beta$-enhanced contributions ensures that terms proportional to $v_{2}$ are kept thanks to the relation $v c_{\beta} \operatorname{tg} \beta=v_{2}$ that are then shifted by the full Higgs field $v_{2} \rightarrow \sqrt{2} \phi_{2}^{0 *}$ according to the discussion of Sect. 2.2.

For the calculation of $\Delta_{b, 2}^{e l w, 2}$ we have to use the offdiagonal propagators of Eq. (22) proportional to the isospin gauge coupling $g$, but have to include chargino propagators for the diagrams involving top squarks in addition. In the basis $\left(\tilde{W}^{+}, \tilde{H}_{1}^{+}, \tilde{H}_{2}^{+}\right)$, the full inverse chargino propagator matrix is given by

$$
\begin{aligned}
& \mathcal{P}^{-1}=\mathcal{P}_{0}^{-1}+\mathcal{D} \\
& \mathcal{P}_{0}^{-1}=\left(\begin{array}{ccc}
\not p-M_{2} & 0 & 0 \\
0 & \not p & -\mu \\
0 & -\mu & \not \supset
\end{array}\right),
\end{aligned}
$$



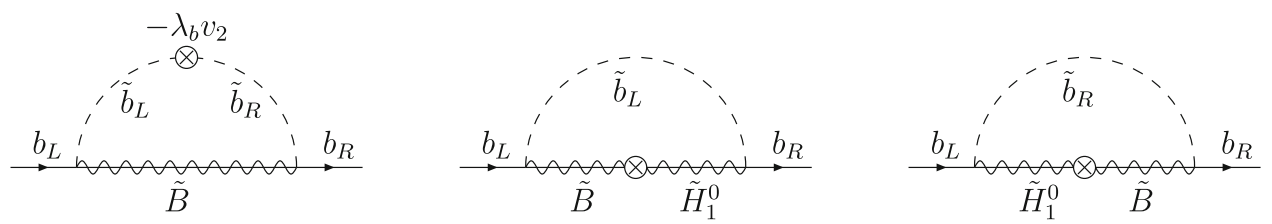

Fig. 3 One-loop diagrams of sbottom-self-energy insertions contributing to the SUSY-QCD corrections $\Delta_{b, 2}^{\text {elw, } 1}$ of the bottom-quark self-energy involving bottom quarks $b$, bottom squarks $\tilde{b}$, binos $\tilde{B}$ and higgsinos $\tilde{H}_{1}^{0}$. The crossed lines indicate off-diagonal propagator contributions

$$
\mathcal{D}=\left(\begin{array}{ccc}
0 & -\frac{g v}{\sqrt{2}} c_{\beta}-\frac{g v}{\sqrt{2}} s_{\beta} \\
-\frac{g v}{\sqrt{2}} c_{\beta} & 0 & 0 \\
-\frac{g v}{\sqrt{2}} s_{\beta} & 0 & 0
\end{array}\right)
$$

where the term $\mathcal{D}$ is subleading in the heavy-SUSY-particle limit. The propagator matrix is then obtained as

$$
\begin{aligned}
\mathcal{P}= & \mathcal{P}_{0}-\mathcal{P}_{0} \mathcal{D} \mathcal{P}_{0} \\
\mathcal{P}_{0}= & \left(\begin{array}{ccc}
\frac{\not p+M_{2}}{p^{2}-M_{2}^{2}} & 0 & 0 \\
0 & \frac{\not p}{p^{2}-\mu^{2}} & \frac{\mu}{p^{2}-\mu^{2}} \\
0 & \frac{\mu}{p^{2}-\mu^{2}} & \frac{\not p p}{p^{2}-\mu^{2}}
\end{array}\right) \\
& -\mathcal{P}_{0} \mathcal{D} \mathcal{P}_{0} \\
= & \mathcal{N}\left(\begin{array}{cccc}
(\not p+\mu \operatorname{tg} \beta)\left(\not p+M_{2}\right) & \left(\not p+M_{2}\right)(\not p+\mu \operatorname{tg} \beta) & \left(\not p+M_{2}\right)(\not p \operatorname{tg} \beta+\mu) \\
(\not p \operatorname{tg} \beta+\mu)\left(\not p+M_{2}\right) & 0 & 0 \\
& & 0
\end{array}\right)
\end{aligned}
$$

where the normalization factor reads

$$
\mathcal{N}=\frac{g v c_{\beta}}{\sqrt{2}\left(p^{2}-M_{2}^{2}\right)\left(p^{2}-\mu^{2}\right)}
$$

Inserting the corresponding $\operatorname{tg} \beta$-enhanced terms of the offdiagonal propagators into the self-energies of Fig. 4, we obtain the proper expressions for $\Delta_{b, 2}^{e l w, 2}$ of Eq. (6).

The leading $\operatorname{tg} \beta$-enhanced terms of the off-diagonal chargino- and neutralino-propagator-matrix entries have been used for the calculation of the two-loop SUSY-QCD corrections of corrections $\Delta_{b, 2}^{\text {elw,1/2}}$ to the bottom Yukawa couplings in Eq. (6). The relevant two-loop diagrams of the bottom self-energy are depicted in Fig. 5. The technical method of our calculation follows the analysis of Refs.
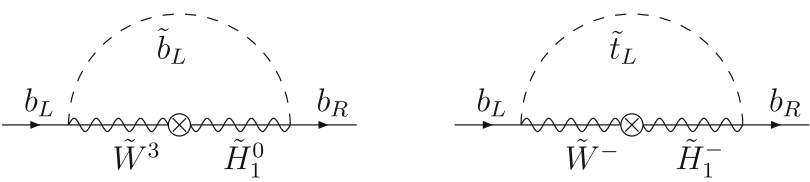

Fig. 4 One-loop diagrams of sbottom-self-energy insertions contributing to the SUSY-QCD corrections $\Delta_{b, 2}^{e l w, 2}$ of the bottom-quark selfenergy involving bottom quarks $b$, sbottoms $\tilde{b}$, stops $\tilde{t}$, winos $\tilde{W}^{3, \pm}$ and higgsinos $\tilde{H}_{1}^{0, \pm}$. The crossed lines indicate the $\operatorname{tg} \beta$-enhanced offdiagonal wino-higgsino propagator contributions
[34,35]. The diagrams of Fig. 5 are evaluated with single crosses in each sbottom/stop or gaugino/higgsino line individually. However, for the additional contributions going with $s_{b}^{2}, c_{b}^{2}, s_{t}^{2}, c_{t}^{2}$ in Eq. (6) diagrams with three crosses have to be taken into account, too, as exemplified for one diagram involving top quarks and stops in Fig. 6. This means that there are contributions with three off-diagonal propagators inserted in the two-loop diagrams. These diagrams complete the corrections to the terms proportional to $s_{b}^{2}, c_{b}^{2}, s_{t}^{2}, c_{t}^{2}$ in Eq. (6) by means of the relations of Eq. (23) and

$M_{L R}^{2}=m_{q}\left(A_{q}-\mu r_{q}\right)=\left(m_{\tilde{q}_{1}}^{2}-m_{\tilde{q}_{2}}^{2}\right) s_{q} c_{q} \quad(q=b, t)$

For the contributions to $\Delta_{b, 2}^{e l w, 2}$, crosses involving the offdiagonal entry of the top-quark propagator matrix

$\mathcal{P}=\frac{1}{p^{2}-m_{t}^{2}}\left(\begin{array}{cc}\not p & m_{t} \\ m_{t} & \not \supset\end{array}\right)$

in the $\left(t_{L}, t_{R}\right)$ basis induced by the large top mass have to be considered, too. It should be noted that the symmetry factors between the self-energies and the effective coupling of the Lagrangian have to be taken into account properly for the shift $v_{2} \rightarrow \sqrt{2} \phi_{2}^{0 *}$.

For the finite result of the two-loop corrections to $\Delta_{b, 2}^{e l w, 1 / 2}$ we have to renormalize the sbottom and stop masses as well as the sbottom and stop mixing angles. The counterterm of the $\Delta_{b, 2}^{e l w, 1}$ term can be derived as

$\delta \Delta_{b, 2, N N L O}^{e l w, 1}=\sum_{i=1,2} \frac{\partial \Delta_{b, 2}^{e l w, 1}}{\partial\left(m_{\tilde{b}_{i}}^{2}\right)} \delta m_{\tilde{b}_{i}}^{2}+\frac{\partial \Delta_{b, 2}^{e l w, 1}}{\partial \theta_{b}} \delta \theta_{b}$

where $\Delta_{b, 2}^{e l w, 1}$ is the NLO expression of Eq. (6). The renormalization constants are given by

$$
\begin{aligned}
\delta \theta_{b}= & -\frac{C_{F}}{4} \frac{\alpha_{s}}{\pi} \Re e\left\{s_{2 \theta_{b}} c_{2 \theta_{b}} \frac{A_{0}\left(m_{\tilde{b}_{2}}\right)-A_{0}\left(m_{\tilde{b}_{1}}\right)}{m_{\tilde{b}_{2}}^{2}-m_{\tilde{b}_{1}}^{2}}\right\} \\
\delta m_{\tilde{b}_{i}}^{2}= & \frac{C_{F}}{4} \frac{\alpha_{s}}{\pi} \Re e\left\{\left(1+c_{2 \theta_{b}}^{2}\right) A_{0}\left(m_{\tilde{b}_{i}}\right)\right. \\
& +s_{2 \theta_{b}}^{2} A_{0}\left(m_{\tilde{b}_{j}}\right)-2 A_{0}\left(m_{\tilde{g}}\right) \\
& -4 m_{\tilde{b}_{i}}^{2} B_{0}\left(m_{\tilde{b}_{i}}^{2} ; 0, m_{\tilde{b}_{i}}\right)+2\left(m_{\tilde{b}_{i}}^{2}\right. \\
& \left.\left.-m_{\tilde{g}}^{2}\right) B_{0}\left(m_{\tilde{b}_{i}}^{2} ; m_{\tilde{g}}, 0\right)\right\} \quad(j \neq i)
\end{aligned}
$$


Fig. 5 Generic two-loop diagrams of the SUSY-QCD contributions to the bottom self-energy involving bottom quarks $b$, sbottoms $\tilde{b}$ and stops $\tilde{t}$, gluons $g$, gluinos $\tilde{g}$, binos $\tilde{B}$, winos $\tilde{W}$ and higgsinos $\tilde{H}_{1}^{0, \pm}$
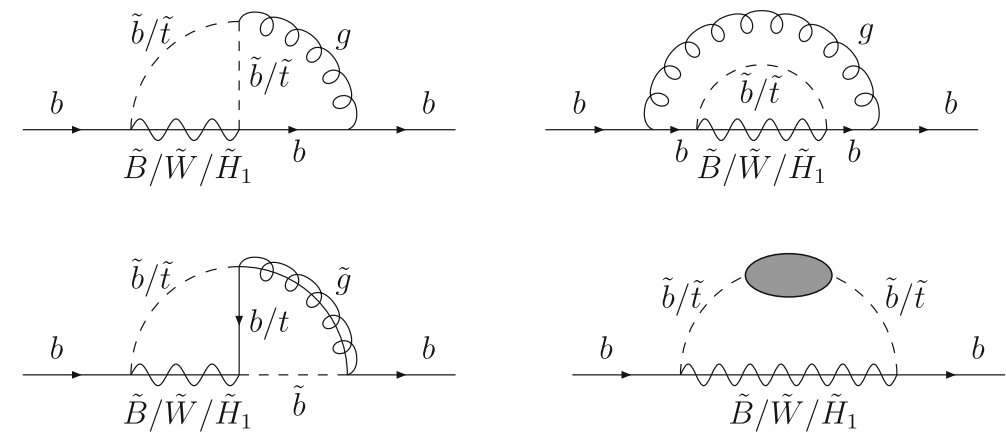

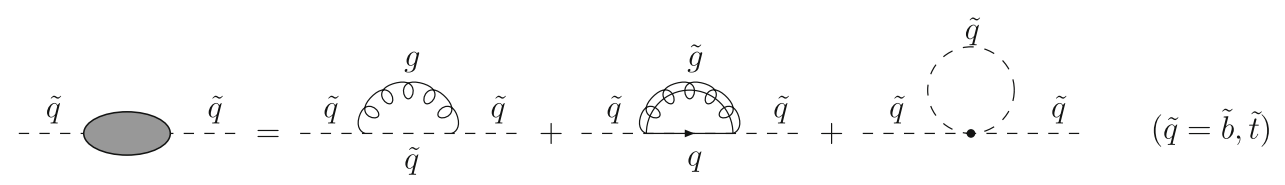

Fig. 6 All possible cross insertions into one of the diagrams contributing to the leading terms of $\Delta_{b, 2}^{e l w, 2}$ at NNLO involving top quarks and stops

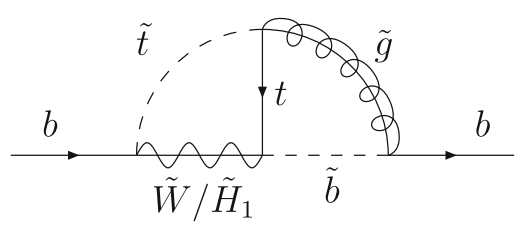

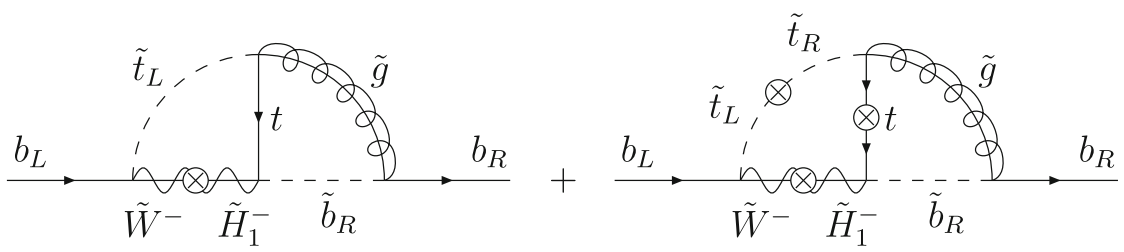

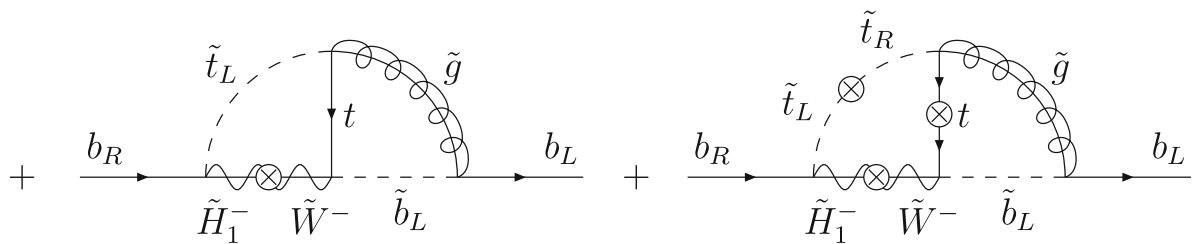

where we neglected kinematical $\mathcal{O}\left(m_{b}\right)$ terms consistently but kept the full mixing-angle dependence. The scalar integrals are defined as

$$
\begin{aligned}
A_{0}(m)=\frac{(4 \pi)^{2}}{i} & \bar{\mu}^{4-n} \int \frac{d^{n} k}{(2 \pi)^{n}} \frac{1}{k^{2}-m^{2}} \\
B_{0}\left(p^{2} ; m_{1}, m_{2}\right)= & \frac{(4 \pi)^{2}}{i} \bar{\mu}^{4-n} \\
& \times \int \frac{d^{n} k}{(2 \pi)^{n}} \frac{1}{\left(k^{2}-m_{1}^{2}\right)\left[(k+p)^{2}-m_{2}^{2}\right]}
\end{aligned}
$$

where $\bar{\mu}$ deotes the 't Hooft mass.

For the $\Delta_{b, 2}^{e l w, 2}$ term we have to renormalize the stop contributions in addition so that the full counterterm is given by

$$
\begin{aligned}
\delta \Delta_{b, 2, N N L O}^{e l w, 2}= & \sum_{i=1,2} \frac{\partial \Delta_{b, 2}^{e l w, 2}}{\partial\left(m_{\tilde{b}_{i}}^{2}\right)} \delta m_{\tilde{b}_{i}}^{2}+\frac{\partial \Delta_{b, 2}^{e l w, 2}}{\partial \theta_{b}} \delta \theta_{b} \\
& +\sum_{i=1,2} \frac{\partial \Delta_{b, 2}^{e l w, 2}}{\partial\left(m_{\tilde{t}_{i}}^{2}\right)} \delta m_{\tilde{t}_{i}}^{2}+\frac{\partial \Delta_{b, 2}^{e l w, 2}}{\partial \theta_{t}} \delta \theta_{t}
\end{aligned}
$$

with the NLO contribution $\Delta_{b, 2}^{\text {elw,2 }}$ of Eq. (6), the renormalization constants $\delta m_{\tilde{b}_{i}}^{2}, \delta \theta_{b}$ of Eq. (30) and

$$
\begin{aligned}
\delta \theta_{t}= & \frac{C_{F}}{4} \frac{\alpha_{s}}{\pi} c_{2 \theta_{t}} \Re e\left\{s_{2 \theta_{t}} \frac{A_{0}\left(m_{\tilde{t}_{2}}\right)-A_{0}\left(m_{\tilde{t}_{1}}\right)}{m_{\tilde{t}_{1}}^{2}-m_{\tilde{t}_{2}}^{2}}\right. \\
& \left.+2 m_{\tilde{g}} m_{t} \frac{B_{0}\left(m_{\tilde{t}_{1}}^{2} ; m_{\tilde{g}}, m_{t}\right)+B_{0}\left(m_{\tilde{t}_{2}}^{2} ; m_{\tilde{g}}, m_{t}\right)}{m_{\tilde{t}_{1}}^{2}-m_{\tilde{t}_{2}}^{2}}\right\}
\end{aligned}
$$




$$
\begin{aligned}
\delta m_{\tilde{t}_{i}}^{2}= & \frac{C_{F}}{4} \frac{\alpha_{s}}{\pi} \Re e\left\{\left(1+c_{2 \theta_{t}}^{2}\right) A_{0}\left(m_{\tilde{t}_{i}}\right)+s_{2 \theta_{t}}^{2} A_{0}\left(m_{\tilde{t}_{j}}\right)\right. \\
& -2 A_{0}\left(m_{\tilde{g}}\right)-2 A_{0}\left(m_{t}\right) \\
& -4 m_{\tilde{t}_{i}}^{2} B_{0}\left(m_{\tilde{t}_{i}}^{2} ; 0, m_{\tilde{t}_{i}}\right) \\
& \left.-2\left(m_{\tilde{g}}^{2}+m_{t}^{2}-m_{\tilde{t}_{i}}^{2} \mp m_{\tilde{g}} m_{t} s_{2 \theta_{t}}\right) B_{0}\left(m_{\tilde{b}_{i}}^{2} ; m_{\tilde{g}}, m_{t}\right)\right\} \quad(j \neq i)
\end{aligned}
$$

for the stop renormalization constants, where we kept all terms proportional to the top mass $m_{t}$. The final results have been explicitly checked to be ultraviolet finite after renormalization. The whole calculation has been performed twice independently with different methods and implementations.

Due to the mismatch between the $(n-2)$ gluonic d.o.f. and the 2 d.o.f. of the gluinos in dimensional regularization, anomalous counterterms have to be added to restore supersymmetry [66]. This affects the SUSY-counterparts $\hat{g}, \hat{g}^{\prime}$ of the electroweak gauge couplings at the $\tilde{B} q \tilde{q}$ and $\tilde{W} q \tilde{q}$ vertices $(q=t, b$ and $\tilde{q}=\tilde{b}, \tilde{t})$,

$\hat{g}=g\left[1-\frac{C_{F}}{8} \frac{\alpha_{s}}{\pi}\right], \quad \hat{g}^{\prime}=g^{\prime}\left[1-\frac{C_{F}}{8} \frac{\alpha_{s}}{\pi}\right]$

as well as the SUSY-counterparts of the Higgs Yukawa couplings $^{6}$,

$\lambda_{\tilde{H} q \tilde{q}}=\lambda_{H q q}\left[1-\frac{3}{8} C_{F} \frac{\alpha_{s}}{\pi}\right]$,

$\lambda_{H \tilde{q} \tilde{q}}=\lambda_{H q q}\left[1-\frac{C_{F}}{4} \frac{\alpha_{s}}{\pi}\right]$

This results in anomalous counterterms of the contributions $\Delta_{b, 2}^{e l w, 1 / 2}$,

$\delta \Delta_{b, 2, \text { anom }}^{e l w, 1}=-\frac{C_{F}}{2} \frac{\alpha_{s}}{\pi} \Delta_{b, 2}^{e l w, 1}$

$\delta \Delta_{b, 2, \text { anom }}^{\text {elw,2 }}=-\frac{C_{F}}{2} \frac{\alpha_{s}}{\pi} \Delta_{b, 2}^{\text {elw }, 2}$

where $\Delta_{b, 2}^{e l w, 1 / 2}$ denote the one-loop expressions of Eq. (6).

\subsection{Strange Yukawa couplings}

The translation of the results for the bottom-Yukawa couplings to the Higgs boson couplings to strange quarks requires a careful investigation of the corresponding quarkmass contributions. Since in the calculation of the bottomYukawa coupling the bottom quark is treated strictly massless and the external momentum dependence is omitted, there is no difference for the individual two-loop diagrams, if the bottom parameters are replaced by their corresponding strange parameters. Care must be taken for the proper summation

\footnotetext{
6 The same anomalous counterterms arise for the charged higgsino coupling $\lambda_{H^{ \pm} \tilde{t} b}$, too.
}

over all quark/squark flavours for the diagrams with gluinoself-energy insertions since the strange-squark mass coincides with the left- and right-handed squark masses of the second generation and the sbottom and stop masses of the third generation are independent. Another difference to the bottom-quark case is the absence of sizeable charm-Yukawainduced SUSY-electroweak contributions to the strangeYukawa coupling, since we are neglecting the charm-Yukawa coupling $\lambda_{c}$. The final result can be cast into the form

$$
\Delta_{s}=\frac{\Delta_{s, 2}^{Q C D}\left[1+\delta_{s}\right]+\Delta_{s, 2}^{e l w, 1}\left[1+\delta_{1}\right]+\Delta_{s, 2}^{e l w, 2}\left[1+\delta_{2}\right]}{1+\Delta_{s, 1}\left[1+\delta_{s}\right]} \operatorname{tg} \beta
$$

where $\delta_{S}\left(\delta_{1 / 2}\right)$ denotes the NNLO SUSY-QCD corrections to the QCD (electroweak) part of the strange Yukawa couplings and $\Delta_{s, 1 / 2}^{Q C D}, \Delta_{s, 2}^{e l w, 1 / 2}$ are defined in Eq. (7). The expression above for $\Delta_{S}$ is then inserted into the effective Lagrangian of Eq. (5) and into the resummed couplings of Eq. (13), respectively, resumming in this way all terms of $\mathcal{O}\left[\left(\Delta_{s}\right)^{n}\right]$.

\section{Results}

The final results of this paper have been implemented in the program Hdecay [68-70]. This code computes the MSSM Higgs couplings and masses based on the RG-improved expressions of Ref. [71]. Moreover, the partial decay widths and branching ratios of the MSSM Higgs bosons are calculated including higher-order corrections [36-38]. For large $\operatorname{tg} \beta$ values the dominant neutral Higgs boson decays are into $b \bar{b}$ and $\tau^{+} \tau^{-}$. In Ref. [39] their branching ratios have been analyzed including the correction $\Delta_{b}$ of Eq. (6) at the oneloop level.

\subsection{Higgs decays into bottom and strange quarks}

The QCD and SUSY-QCD corrected partial decay widths of the neutral Higgs bosons $\Phi=h, H, A$ into bottom quarks can be expressed as [39]

$$
\begin{aligned}
\Gamma[\Phi & \rightarrow b \bar{b}]=\frac{3 G_{F} M_{\Phi}}{4 \sqrt{2} \pi} \bar{m}_{b}^{2}\left(M_{\Phi}\right) \\
& \times\left[1+\delta_{\mathrm{QCD}}+\delta_{t}^{\Phi}\right] \tilde{g}_{b}^{\Phi}\left[\tilde{g}_{b}^{\Phi}+\delta_{S Q C D}^{r e m}\right]
\end{aligned}
$$

with $\bar{m}_{b}\left(M_{\Phi}\right)$ denoting the $\overline{\mathrm{MS}}$ bottom mass at the scale of the Higgs mass $M_{\Phi}$ and quark mass effects beyond $\mathcal{O}\left(m_{b}^{2}\right)$ are neglected. The QCD corrections $\delta_{\mathrm{QCD}}$ and the top quark induced contributions $\delta_{t}^{\Phi}$ are known [72-86] and can be found in Refs. [36-38] in compact form. The QCD corrections $\delta_{\mathrm{QCD}}$ are taken into account up to $\mathrm{N}^{4} \mathrm{LO}$ and the corrections $\delta_{t}^{\Phi}$ at the NNLO level in Hdecay. 
The leading contributions of the SUSY-QCD corrections $[87,88]$ have been absorbed in the resummed bottom-Yukawa couplings $\tilde{g}_{b}^{\phi}$ as given in Eq. (13). The remainder $\delta_{S Q C D}^{r e m}$ is small, i.e. at the sub-per-cent level, in phenomenologically relevant scenarios for large $\operatorname{tg} \beta$ values [39]. This observation at NLO implies the expectation that at higher orders the remainders after factorizing the corrections involved in the effective Lagrangian of Eq. (12) are even smaller and thus negligible in general. This underlines that the results of our work constitute the major part of the corrections beyond NLO with tiny remainders at higher loop-levels. It should be noted that our two-loop corrections to the $A_{b}, A_{s}$-induced terms are formally of three-loop order of the related physical observables involving the corresponding effective Yukawa couplings, but they modify the relations between the Yukawa couplings and quark masses at NNLO. In our analysis we include the full (two-loop corrected) $\Delta_{b}\left(\Delta_{S}\right)$ contributions including the QCD and electroweak parts in the couplings $\tilde{g}_{b}^{\phi}\left(\tilde{g}_{s}^{\phi}\right)$.

The strange Yukawa coupling plays a phenomenological role for charged Higgs decays into charm and strange quarks $H^{+} \rightarrow c \bar{s}$. Neglecting regular quark mass effects ${ }^{7}$ this partial decay width can be expressed as $[89-91]^{8}$

$$
\begin{aligned}
\Gamma[ & \left.H^{+} \rightarrow c \bar{s}\right]=\frac{3 G_{F} M_{H^{ \pm}}}{4 \sqrt{2} \pi}\left|V_{c s}\right|^{2} \\
& \times\left[\bar{m}_{c}^{2}\left(M_{H^{ \pm}}\right)\left(g_{c}^{A}\right)^{2}+\bar{m}_{s}^{2}\left(M_{H^{ \pm}}\right)\left(\tilde{g}_{s}^{A}\right)^{2}\right]\left(1+\delta_{\mathrm{QCD}}\right)
\end{aligned}
$$

with the same QCD-correction-factor $\delta_{Q C D}$ as in Eq. (38). The small remainder of the genuine SUSY-QCD corrections after absorbing the dominant part in the effective strangeYukawa coupling $\tilde{g}_{s}^{A}$ is neglected.

\subsection{Numerical results}

We perform our numerical analysis of the MSSM Higgs boson decays into bottom and strange quarks for the MSSM benchmark scenario $M_{h}^{125}$ [32] as a representative case ${ }^{9}$ :

$$
\begin{aligned}
M_{h}^{125}: \operatorname{tg} \beta & =40, \quad M_{\tilde{Q}}=1.5 \mathrm{TeV}, \\
M_{\tilde{\ell}_{3}} & =2 \mathrm{TeV}, \quad M_{\tilde{g}}=2.5 \mathrm{TeV}, \\
M_{1} & =M_{2}=1 \mathrm{TeV},
\end{aligned}
$$

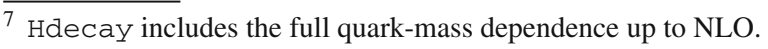

${ }^{8} V_{c s}$ denotes the corresponding CKM-matrix element.

9 The values for $A_{b}, A_{\tau}, A_{t}$ have been obtained from $X_{t}=A_{t}-$ $\mu / \operatorname{tg} \beta=2.8 \mathrm{TeV}$. The trilinear coupling $A_{s}$ has been chosen as $A_{s}=$ $A_{b}$. The soft SUSY-breaking squark mass parameter $M_{\tilde{Q}}$ is defined in the on-shell scheme. We have determined the corresponding $\overline{\mathrm{MS}}$ parameters by appropriate iterations. The $\overline{\mathrm{MS}}$ squark-mass parameters of the second generation have been identified with the corresponding ones of the third generation.
}

$$
A_{b}=A_{\tau}=A_{t}=2.825 \mathrm{TeV}, \quad \mu=1 \mathrm{TeV}
$$

For the Higgs masses and couplings we use the RG-improved two-loop expressions of Ref. [71], so that the leading corrections at the one- and two-loop level to the Higgs masses and the effective mixing angle $\alpha$ are taken into account. The bottom $\overline{\mathrm{MS}}$-mass has been chosen as $\overline{m_{b}}\left(\overline{m_{b}}\right)=4.18 \mathrm{GeV}$, which corresponds to a pole mass $m_{b}=4.84 \mathrm{GeV}$ according to the implementation in Hdecay that determines the bottom-quark pole mass from the $\overline{\mathrm{MS}}$-mass at the scale of the pole mass. The strange $\overline{\mathrm{MS}}$-mass has been initialized as $\overline{m_{s}}(2 \mathrm{GeV})=95 \mathrm{MeV}$ and the strong coupling constant as $\alpha_{s}\left(M_{Z}\right)=0.118$. The top pole mass has been taken to be $m_{t}=172.5 \mathrm{GeV}$. In the mass matrices of the stop and sbottom states effective top and bottom masses are implemented as discussed in Ref. [67]. With this set-up we obtain the following squark masses e.g. for $\operatorname{tg} \beta=40$,

$$
\begin{array}{ll}
m_{\tilde{t}_{1}}=1374.8 \mathrm{GeV}, & m_{\tilde{t}_{2}}=1632.6 \mathrm{GeV}, \\
m_{\tilde{b}_{1}}=1479.3 \mathrm{GeV}, & m_{\tilde{b}_{2}}=1525.3 \mathrm{GeV}, \\
m_{\tilde{c}_{1}}=1502.6 \mathrm{GeV}, & m_{\tilde{c}_{2}}=1503.1 \mathrm{GeV}, \\
m_{\tilde{s}_{1}}=1500.2 \mathrm{GeV}, & m_{\tilde{s}_{2}}=1504.8 \mathrm{GeV}
\end{array}
$$

We will present the impact of the new results on the bottom- and strange-Yukawa couplings as well as related observables. Figure 7 displays the scale dependence of the SUSY-QCD parts of $\Delta_{b}$ and $\Delta_{s}$,

$\Delta_{q}^{Q C D}=\frac{\Delta_{q, 2}^{Q C D}\left(1+\delta_{q}\right)}{1+\Delta_{q, 1}\left(1+\delta_{q}\right)} \operatorname{tg} \beta \quad(q=b, s)$

with and without the $A_{b}, A_{s}$ contributions $\Delta_{b, 1}, \Delta_{s, 1}$ for the $M_{h}^{125}$ scenario. For $\operatorname{tg} \beta=40$ the $\Delta_{b}$ and $\Delta_{s}$ corrections range at the level of $30 \%$. The scale dependence is reduced significantly from one- to two-loop order to a few per cent at NNLO, while the additional contributions of the $A_{b}, A_{s}$ terms are small, i.e. at the per-cent level, as can be inferred from the differences between the red and blue curves. The comparison between the blue and red curves shows that the additional scale dependence induced by $A_{b}$ and $A_{s}$ is numerically small in line with neglecting terms that are not of $\mathcal{O}(\mu \operatorname{tg} \beta)$ or $\mathcal{O}\left(A_{b, s}\right)$. The effects of the contributions induced by $A_{b}, A_{s}$ are significantly larger than the NLO remainder so that this can naturally be expected for the remainders at NNLO and beyond, too. However, in general the sizes and directions of the total $\Delta_{b}$ and $\Delta_{s}$ corrections depend on the MSSM scenario, in particular on the sign and size of $\mu$ and the value of $\operatorname{tg} \beta$. The central scales have been chosen equal to the average of the corresponding SUSY masses, i.e. $\mu_{0}=\left(m_{\tilde{q}_{1}}+m_{\tilde{q}_{2}}+m_{\tilde{g}}\right) / 3$ for $\Delta_{b, 1 / 2}^{Q C D}, \Delta_{s, 1 / 2}^{Q C D}$. For the electroweak parts we have adopted $\mu_{0}=\left(m_{\tilde{t}_{1}}+m_{\tilde{t}_{2}}+\mu\right) / 3$ for $\Delta_{b, 2}^{e l w, t}$ and the average of the involved SUSY particles for the strong-coupling scale involved in the individual con- 

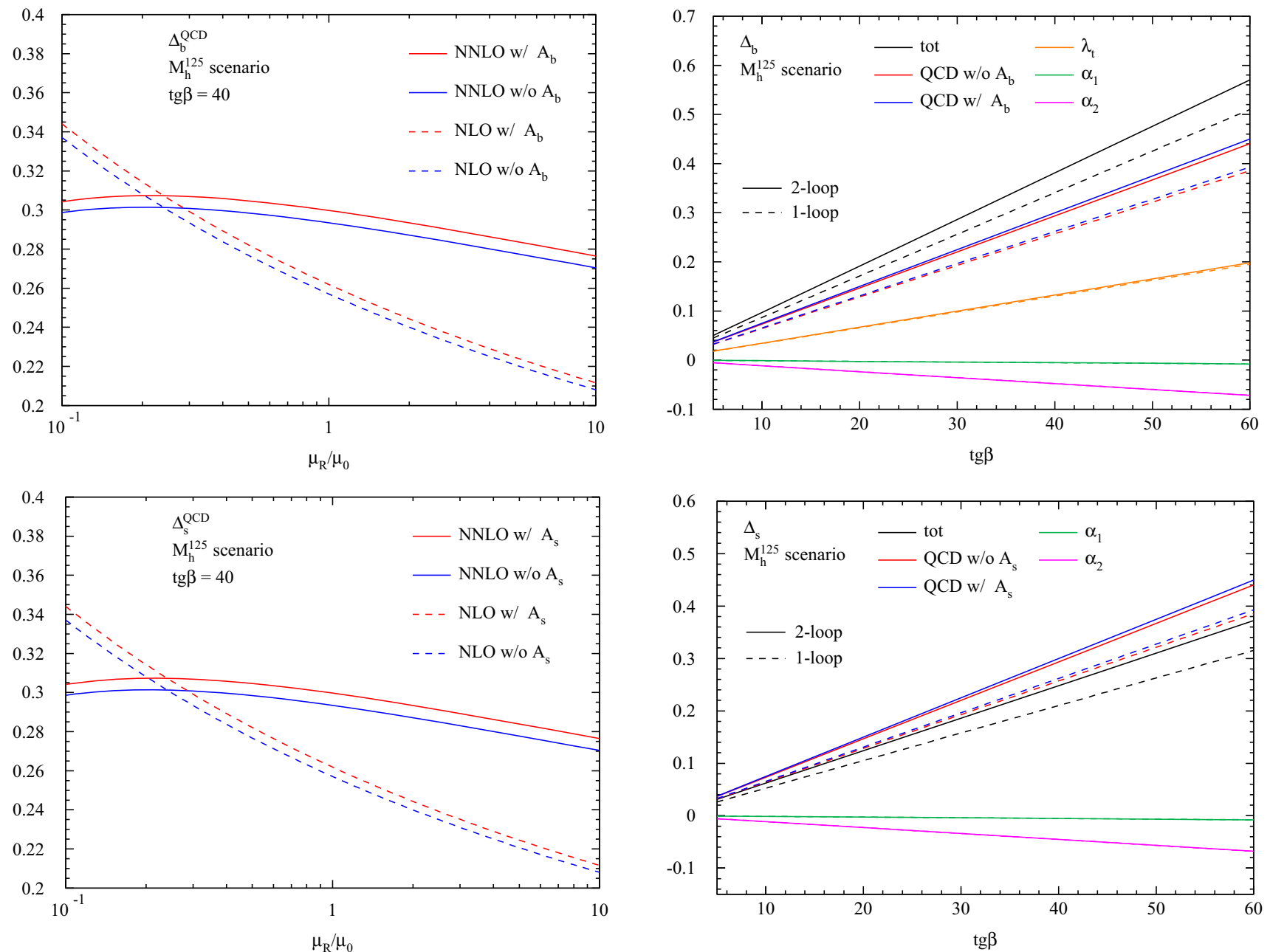

Fig. 7 Scale dependence of the SUSY-QCD corrections $\Delta_{b}^{Q C D}$ (upper plot) and $\Delta_{s}^{Q C D}$ (lower plot) at one-loop and two-loop order with and without the $A_{b}, A_{s}$ contributions in the $M_{h}^{125}$ scenario

tributions to $\Delta_{q, 2}^{e l w, 1 / 2}(q=b, s)$ at NNLO. These choices are suitable as the appropriate natural central scales.

In Fig. 8 we present the individual and total contributions to $\Delta_{b}$ and $\Delta_{s}$ as a function of $\operatorname{tg} \beta$ for the $M_{h}^{125}$ scenario. ${ }^{10}$ While the SUSY-QCD parts $\Delta_{b / s, 2}^{Q C D}$ dominate the contributions, the electroweak contributions $\Delta_{b / s, 2}^{e l w, t / 2}$ amount to $\mathcal{O}(10 \%)$ reaching up to about $20 \%$ for large $\operatorname{tg} \beta$-values. Especially the terms $\Delta_{b / s, 2}^{e l w, t}$ cannot be neglected if a prediction with an accuracy at the per-cent level should be achieved. The two-loop corrections to the electroweak terms range accidentially at the per-cent level in this benchmark scenario ${ }^{11}$. They

\footnotetext{
The values of $A_{t}=A_{b}=A_{\tau}$ have been derived from $X_{t}=2.8 \mathrm{TeV}$ for each value of $\operatorname{tg} \beta$ accordingly.

${ }^{11}$ For the $\Delta_{b, 2}^{\text {elw,t }}$ terms they amount to about $1.7 \%$, for $\Delta_{b, 2}^{\text {elw,1 }}$ to about $0.4 \%$ and for $\Delta_{b, 2}^{e l w, 2}$ to about $-0.7 \%$.
}

Fig. 8 Dependence of the full SUSY-QCD + SUSY-electroweak corrections $\Delta_{b}$ (upper plot) and $\Delta_{s}$ (lower plot) on $\operatorname{tg} \beta$ at one-loop and two-loop order with and without the $A_{b}, A_{s}$ contributions in the $M_{h}^{125}$ scenario, and the individual contributions to $\Delta_{b}$ and $\Delta_{s}$

can be larger in other scenarios reaching a level of $10-15 \%$ in general.

To exemplify this, we show the size of the two-loop corrections to the terms $\Delta_{b, s}^{\text {elw, } 1 / 2}$ in Fig. 9 as a function of the gaugino masses $M=M_{1}=M_{2}$ with all other MSSM parameters kept fixed at their values of the $M_{h}^{125}$ scenario. For small gaugino masses the corrections can amount to more than $20 \%$.

As a particular application we analyze the partial decay widths of the heavy neutral MSSM Higgs bosons into $b \bar{b}$ pairs and of the charged Higgs boson into $c \bar{s}$ in Fig. 10 for the $M_{h}^{125}$ scenario, respectively. The two-loop parts of $\Delta_{b}$ $\left(\Delta_{s}\right)$ reduce the partial decay widths to $b \bar{b}(c \bar{s})$ pairs for the central scale choices by $\mathcal{O}(10 \%)$. The NLO bands (dashed blue curves) and the NNLO bands (full red curves) are generated by varying the renormalization scales of $\Delta_{b}$ and $\Delta_{s}$ between $1 / 3$ and 3 times the corresponding central scales 

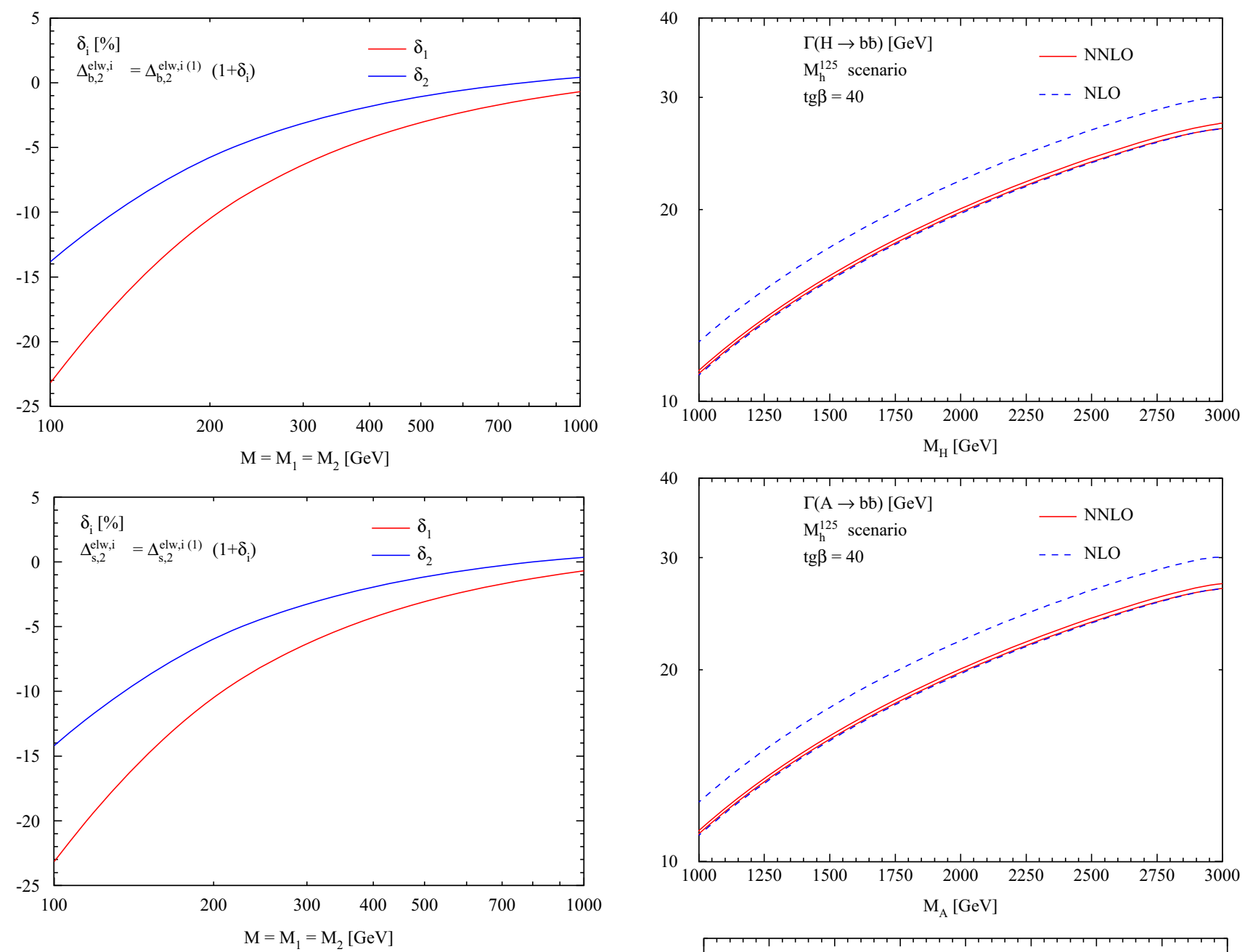

Fig. 9 Radiative corrections to the SUSY-electroweak corrections $\Delta_{b}^{e l w, 1 / 2}$ (upper plot) and $\Delta_{s}^{e l w, 1 / 2}$ (lower plot) as a function of the gaugino mass parameters $M=M_{1}=M_{2}$

$\mu_{0} .{ }^{12}$ A significant reduction of the dashed one-loop bands of $\mathcal{O}(10 \%)$ to the full two-loop bands at the per-cent level can be inferred from these results which is expected based on the previously known two-loop corrections [34,35]. All NNLO results are positioned at the lower ends of the NLO error bands.

\section{Conclusions}

We have calculated the NNLO corrections to the effective bottom- and strange-quark-Yukawa couplings within the MSSM, extending previous analyses to non-leading terms that are mediated by the soft SUSY-breaking trilinear cou-

\footnotetext{
12 We have extended the usual range to a factor of three, because for a factor of two, the bands do not overlap so that the more restricted range is not appropriate.
}

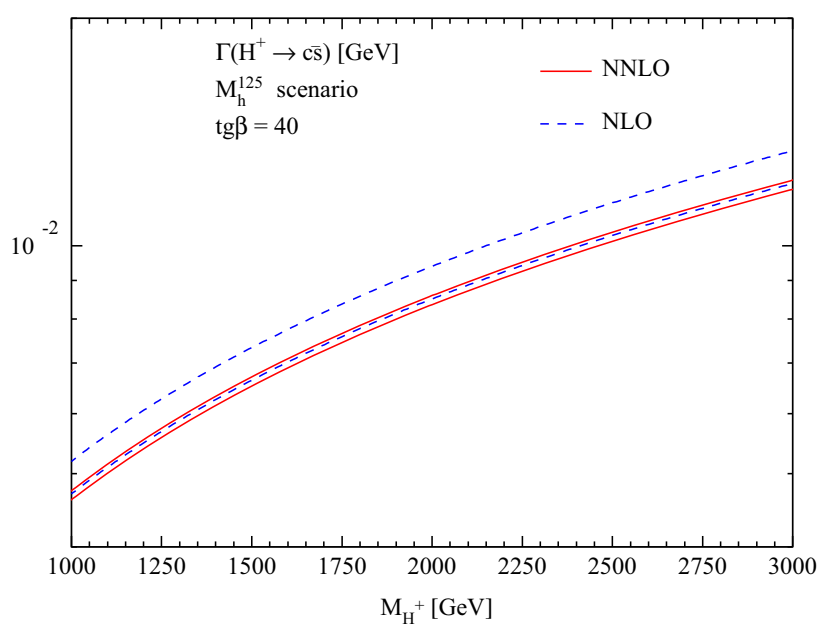

Fig. 10 Partial decay widths of the heavy scalar $H$ and the pseudoscalar $A$ Higgs bosons to $b \bar{b}$ (upper two plots) and charged Higgs decays to $c \bar{s}$ (lower plot) in the $M_{h}^{125}$ scenario. The dashed blue bands display the scale dependence at the one-loop level and the full red bands at the two-loop level by varying the renormalization scales of $\Delta_{b}$ and $\Delta_{s}$ between $1 / 3$ and 3 times the central scale fixed by the average of the involved SUSY-particle masses 
plings $A_{b}, A_{s}$ and the weak couplings $\alpha_{1}, \alpha_{2}$ for large values of $\operatorname{tg} \beta$. The dominant contributions of the SUSYQCD corrections arise from virtual two-loop squark and gluino exchange, that factorize and can be absorbed in effective Yukawa couplings. We have derived the two-loop SUSY-QCD corrections to the effective bottom- and strangeYukawa couplings beyond the leading contributions obtained previously.

In summary, the significant scale dependence of $\mathcal{O}(10 \%)$ of the NLO predictions for processes involving the bottomand strange-quark-Yukawa couplings of MSSM Higgs bosons necessitate the inclusion of NNLO corrections. For the NNLO-corrected Yukawa couplings, we observe a reduction of the scale dependence to the per-cent level. These results were previously known for the leading terms of the bottom-Yukawa couplings. In this work they have been established for the non-leading $A_{b}$ and electroweak terms and the strange-Yukawa couplings. The improved NNLO results for the bottom- and strange-Yukawa couplings provide a quantitative basis for experimental analyses at the LHC and future $e^{+} e^{-}$colliders as the ILC.

Acknowledgements The authors are indebted to M. Mühlleitner for carefully reading the manuscript and very useful comments. This work is supported in part by the Swiss National Science Foundation.

Data Availability Statement This manuscript has no associated data or the data will not be deposited [Authors' comment: Since it describes an analytical calculation and there is no external data in the manuscript.]

Open Access This article is licensed under a Creative Commons Attribution 4.0 International License, which permits use, sharing, adaptation, distribution and reproduction in any medium or format, as long as you give appropriate credit to the original author(s) and the source, provide a link to the Creative Commons licence, and indicate if changes were made. The images or other third party material in this article are included in the article's Creative Commons licence, unless indicated otherwise in a credit line to the material. If material is not included in the article's Creative Commons licence and your intended use is not permitted by statutory regulation or exceeds the permitted use, you will need to obtain permission directly from the copyright holder. To view a copy of this licence, visit http://creativecomm ons.org/licenses/by/4.0/.

Funded by SCOAP ${ }^{3}$.

\section{References}

1. G. Aad et al., [ATLAS Collaboration]. Phys. Lett. B 716, 1 (2012)

2. S. Chatrchyan et al., [CMS Collaboration]. Phys. Lett. B 716, 30 (2012)

3. P.W. Higgs, Phys. Lett. 12, 132 (1964)

4. P.W. Higgs, Phys. Rev. Lett. 13, 508 (1964)

5. P.W. Higgs, Phys. Rev. 145, 1156 (1966)

6. F. Englert, R. Brout, Phys. Rev. Lett. 13, 321 (1964)

7. G.S. Guralnik, C.R. Hagen, T.W. Kibble, Phys. Rev. Lett. 13, 585 (1964)

8. T.W.B. Kibble, Phys. Rev. 155, 1554 (1967)

9. G’t Hooft, Nucl. Phys. B 35, 167 (1971)
10. G't Hooft, M.J.G. Veltman, Nucl. Phys. B 44, 189 (1972)

11. G. Aad et al., [ATLAS and CMS Collaborations]. JHEP 1608, 045 (2016)

12. E. Gildener, S. Weinberg, Phys. Rev. D 13, 3333 (1976)

13. S. Weinberg, Phys. Rev. D 13, 974 (1976)

14. S. Weinberg, Phys. Rev. D 19, 1277 (1979)

15. L. Susskind, Phys. Rev. D 20, 2619 (1979)

16. Y.A. Golfand, E.P. Likhtman, JETP Lett. 13, 323 (1971), [Pisma Zh. Eksp. Teor. Fiz. 13 (1971) 452]

17. D.V. Volkov, V.P. Akulov, Phys. Lett. 46B, 109 (1973)

18. J. Wess, B. Zumino, Nucl. Phys. B 70, 39 (1974)

19. P. Fayet, S. Ferrara, Phys. Rep. 32, 249 (1977)

20. H.P. Nilles, Phys. Rept. 110, 1 (1984)

21. R. Barbieri, Riv. Nuovo Cim. 11N4, 1 (1988)

22. H.E. Haber, G.L. Kane, Phys. Rep. 117, 75 (1985)

23. P. Draper, G. Lee, C.E.M. Wagner, Phys. Rev. D 89(5), 055023 (2014)

24. E. Bagnaschi, G.F. Giudice, P. Slavich, A. Strumia, JHEP 1409, 092 (2014)

25. K. Cheung, R. Huo, J.S. Lee, Y.L. Sming Tsai, JHEP 1504, 151 (2015)

26. J. Pardo Vega, G. Villadoro, JHEP 1507, 159 (2015)

27. H. Bahl, W. Hollik, Eur. Phys. J. C 769, 499 (2016)

28. P. Slavich et al., arXiv:2012.15629 [hep-ph]

29. S.P. Martin, Phys. Rev. D 75, 055005 (2007)

30. R.V. Harlander, P. Kant, L. Mihaila, M. Steinhauser, Phys. Rev. Lett. 100, 191602, (2008) [Erratum ibid. 101, 039901 (2008)]

31. R.V. Harlander, J. Klappert, A. Voigt, Eur. Phys. J. C 77(12), 814 (2017)

32. E. Bagnaschi et al., Eur. Phys. J. C 79(7), 617 (2019)

33. G. Aad et al., [ATLAS Collaboration]. JHEP 1603, 127 (2016)

34. D. Noth, M. Spira, Phys. Rev. Lett. 101, 181801 (2008)

35. D. Noth, M. Spira, JHEP 1106, 084 (2011)

36. M. Spira, Fortsch. Phys. 46, 203 (1998)

37. M. Spira, Prog. Part. Nucl. Phys. 95, 98 (2017)

38. A. Djouadi, Phys. Rep. 459, 1 (2008)

39. J. Guasch, P. Häfliger, M. Spira, Phys. Rev. D 68, 115001 (2003)

40. M. Carena, D. Garcia, U. Nierste, C.E. Wagner, Nucl. Phys. B 577, 88 (2000)

41. P. Häfliger, M. Spira, Nucl. Phys. B 719, 35 (2005)

42. P. Häfliger, M. Spira, PoS HEP2005, 351 (2006)

43. S. Dittmaier, M. Krämer, A. Mück, T. Schlüter, JHEP 0703, 114 (2007)

44. S. Dawson, C.B. Jackson, P. Jaiswal, Phys. Rev. D 83, 115007 (2011)

45. S. Dittmaier, P. Häfliger, M. Krämer, M. Spira, M. Walser, Phys, Rev. D 90(3), 035010 (2014)

46. P. Häfliger, PhD thesis, ETH (Zürich) DISS-ETH-16970, (2006)

47. M. Walser, PhD thesis, ETH (Zürich) DISS-ETH-17592, (2008)

48. L.J. Hall, R. Rattazzi, U. Sarid, Phys. Rev. D 50, 7048 (1994)

49. R. Hempfling, Phys. Rev. D 49, 6168 (1994)

50. M. Carena, M. Olechowski, S. Pokorski, C.E. Wagner, Nucl. Phys. B 426, 269 (1994)

51. J.R. Ellis, M.K. Gaillard, D.V. Nanopoulos, Nucl. Phys. B 106, 292 (1976)

52. M.A. Shifman, A.I. Vainshtein, M.B. Voloshin, V.I. Zakharov, Sov. J. Nucl. Phys. 30, 711 (1979), [Yad. Fiz. 30, 1368 (1979)]

53. M. Spira, A. Djouadi, D. Graudenz, P.M. Zerwas, Nucl. Phys. B 453, 17 (1995)

54. B.A. Kniehl, M. Spira, Z. Phys. C 69, 77 (1995)

55. W. Kilian, Z. Phys. C 69, 89 (1995)

56. L. Mihaila, C. Reisser, JHEP 1008, 021 (2011)

57. A. Crivellin, C. Greub, Phys. Rev. D 87, 015013, (2013) Erratum: [Phys. Rev. D87(2013), 079901]

58. L. Mihaila, N. Zerf, JHEP 1705, 019 (2017) 
59. C. Hamzaoui, M. Pospelov, M. Toharia, Phys. Rev. D 59, 095005 (1999)

60. K.S. Babu, C.F. Kolda, Phys. Rev. Lett. 84, 228 (2000)

61. A.J. Buras, P.H. Chankowski, J. Rosiek, L. Slawianowska, Nucl. Phys. B 619, 434 (2001)

62. A.J. Buras, P.H. Chankowski, J. Rosiek, L. Slawianowska, Nucl, Phys. B 659, 3 (2003)

63. G. Isidori, A. Retico, JHEP 0111, 001 (2001)

64. L. Hofer, U. Nierste, D. Scherer, JHEP 0910, 081 (2009)

65. M. Gorbahn, S. Jäger, U. Nierste, S. Trine, Phys. Rev. D 84, 034030 (2011)

66. S.P. Martin, M.T. Vaughn, Phys. Lett. B 318, 331 (1993)

67. E. Accomando, G. Chachamis, F. Fugel, M. Spira, M. Walser, Phys. Rev. D 85, 015004 (2012)

68. A. Djouadi, J. Kalinowski, M. Spira, Comput. Phys. Commun. 108, $56(1998)$

69. A. Djouadi, M.M. Mühlleitner, M. Spira, Acta Phys. Polon. B 38, 635 (2007)

70. A. Djouadi, J. Kalinowski, M. Mühlleitner, M. Spira, Comput. Phys. Commun. 238, 214 (2019)

71. M. Carena, H.E. Haber, S. Heinemeyer, W. Hollik, C.E. Wagner, G. Weiglein, Nucl. Phys. B 580, 29 (2000)

72. E. Braaten, J.P. Leveille, Phys. Rev. D 22, 715 (1980)

73. N. Sakai, Phys. Rev. D 22, 2220 (1980)

74. T. Inami, T. Kubota, Nucl. Phys. B 179, 171 (1981)

75. S.G. Gorishnii, A.L. Kataev, S.A. Larin, Sov. J. Nucl. Phys. 40, 329 (1984), [Yad. Fiz. 40 (1984) 517]

76. M. Drees and K.I. Hikasa, Phys. Rev. D41 (1990) 1547
77. M. Drees and K.I. Hikasa, Phys. Lett. B240 (1990) 455 [Erratumibid. B262 (1991) 497]

78. S.G. Gorishnii, A.L. Kataev, S.A. Larin, L.R. Surguladze, Mod. Phys. Lett. A 5, 2703 (1990)

79. S.G. Gorishnii, A.L. Kataev, S.A. Larin, L.R. Surguladze, Phys. Rev. D 43, 1633 (1991)

80. A.L. Kataev, V.T. Kim, Mod. Phys. Lett. A 9, 1309 (1994)

81. L.R. Surguladze, Phys. Lett. B 341, 60 (1994)

82. K.G. Chetyrkin, J.H. Kühn, A.K. Kwiatkowski, Proceedings of the Workshop "QCD at LEP”, Aachen, (1994), arXiv:hep-ph/9407271

83. K.G. Chetyrkin, Phys. Lett. B 390, 309 (1997)

84. K.G. Chetyrkin, A. Kwiatkowski, Nucl. Phys. B 461, 3 (1996)

85. K. Melnikov, Phys. Rev. D 53, 5020 (1996)

86. S.A. Larin, T. van Ritbergen, J.A.M. Vermaseren, Phys. Lett. B 362, 134 (1995)

87. J.A. Coarasa, R.A. Jimenez, J. Solà, Phys. Lett. B 389, 312 (1996)

88. H. Eberl, K. Hidaka, S. Kraml, W. Majerotto, Y. Yamada, Phys. Rev. D 62, 055006 (2000)

89. C.S. Li, R.J. Oakes, Phys. Rev. D 43, 855 (1991)

90. A. Mendez, A. Pomarol, Phys. Lett. B 252, 461 (1990)

91. A. Djouadi, P. Gambino, Phys. Rev. D 51, 218 (1995). Erratum: [Phys. Rev. D53 (1996) 4111] 